\title{
COVID-19 Infection Detection from Chest X-Ray Images Using Hybrid Social Group Optimization and Support Vector Classifier
}

\author{
Asu Kumar Singh ${ }^{1}$ (D) Anupam Kumar ${ }^{1}$ D $\cdot$ Mufti Mahmud $^{2} \mathbb{D} \cdot$ M Shamim Kaiser $^{3}$ (D) Akshat Kishore $^{1}$
}

Received: 10 August 2020 / Accepted: 4 February 2021

(c) The Author(s) 2021

\begin{abstract}
A novel strain of Coronavirus, identified as the Severe Acute Respiratory Syndrome-2 (SARS-CoV-2), outbroke in December 2019 causing the novel Corona Virus Disease (COVID-19). Since its emergence, the virus has spread rapidly and has been declared a global pandemic. As of the end of January 2021, there are almost 100 million cases worldwide with over 2 million confirmed deaths. Widespread testing is essential to reduce further spread of the disease, but due to a shortage of testing kits and limited supply, alternative testing methods are being evaluated. Recently researchers have found that chest X-Ray (CXR) images provide salient information about COVID-19. An intelligent system can help the radiologists to detect COVID-19 from these CXR images which can come in handy at remote locations in many developing nations. In this work, we propose a pipeline that uses CXR images to detect COVID-19 infection. The features from the CXR images were extracted and the relevant features were then selected using Hybrid Social Group Optimization algorithm. The selected features were then used to classify the CXR images using a number of classifiers. The proposed pipeline achieves a classification accuracy of 99.65\% using support vector classifier, which outperforms other state-of-the-art deep learning algorithms for binary and multi-class classification.
\end{abstract}

Keywords Computer-aided detection system $\cdot$ Feature reduction $\cdot$ Evolutionary computing $\cdot$ Social group optimization

\section{Introduction}

In December 2019, China saw a sudden increase in pneumonia patients. Initially, the clear cause of this pneumonia remained shrouded in mystery. However, these were soon to be epidemiologically linked to the wet animal wholesale market [1, 2]. China alerted the World Health Organization (WHO) on the December 31 about the odd

Mufti Mahmud

mufti.mahmud@ntu.ac.uk; muftimahmud@gmail.com

Asu Kumar Singh

itsasusingh@gmail.com

Anupam Kumar

anupamkumar@mait.ac.in

1 CSE Department, Maharaja Agrasen Institute of Technology, Delhi, India

2 Department of Computer Science and Medical Technology Innovation Facility, Nottingham Trent University, Clifton, NG11 8NS Nottingham, UK

3 Institute of Information Technology, Jahangirnagar University, Savar, 1342 Dhaka, Bangladesh cases of pneumonia in one of its populous cities, Wuhan, Hubei Province. The novel virus behind all the unusual pneumonia cases was named SARS-CoV-2 by the WHO and was identified to be belonging to the coronavirus family, which caused Severe Acute Respiratory Syndrome (SARS-CoV) and Middle East Respiratory Syndrome (MERS-CoV) outbreaks. Corona-viruses infect birds and mammals including humans and can cause respiratory tract diseases of varying severity [3]. The first major outbreak of a coronavirus was the 2002-2004 SARS outbreak. The outbreak eventually was declared a global epidemic, with a total of 8000 reported cases and a 9\% mortality rate worldwide [4]. The similarities between the viruses causing SARS-CoV and COVID-19 (SARS-CoV-2) are striking. Both viruses have $86 \%$ similar genome sequences that are analogous with SARS-like viruses found in bats, thereby, indicating that both viruses, transmitted from bats to humans at some point [5] as depicted in Fig. 1.

The virus causing COVID-19 is highly transmittable and spreads mainly through coming in contact with respiratory droplets of an infected person. These droplets can penetrate the human body through inhalation or mouth [6]. As of the 


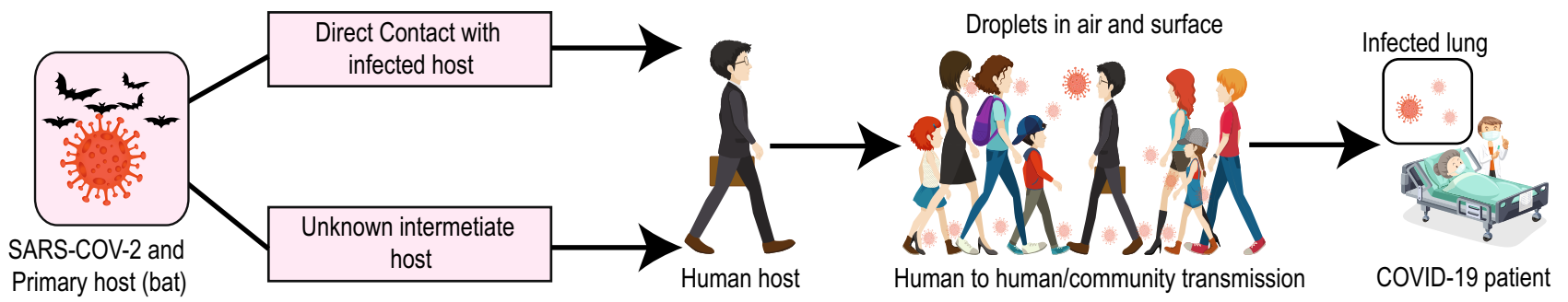

Fig. 1 Block diagram showing SARS-CoV-2 transmission and spreading

end of January 2021, according to statistics of the European Centre for Disease Prevention and Control, there have been almost 100 million confirmed cases worldwide with more than 2 million confirmed deaths as seen in Fig. 2. The mortality rates depend heavily on the age of the patients and prior medical conditions.

The prodrome of COVID-19 generally appears after an incubation period of 5-6 days. The most common symptoms of COVID-19 onset are: fever, cough, and fatigue, while in some cases, the patient may also have a headache, excess sputum, and diarrhea [7]. Particularly, in severe cases, this disease often causes pneumonia leading to oxygen deprivation, thereby damaging the body's vital organs which results in fatal issues such as, kidney failure, heart failure, and other life-threatening complications. But a large number of reported cases show only mild symptoms and can be efficiently treated and managed. Studies reported that the patients with severe symptoms tend to be older in age and had multiple comorbidities such as cardiovascular, digestive or respiratory diseases [8].

The outbreak has caused a major stir worldwide and has led to strict lockdown and social distancing measures being implemented in every affected country. Even developed countries find it difficult to cope with the ongoing demand for intensive care units which are essential to support patients with severe cases of COVID-19. There is also a

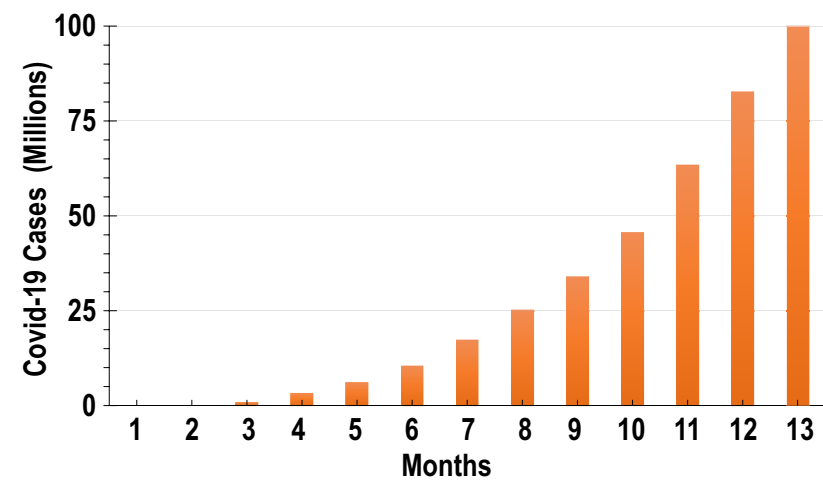

Fig. 2 Number of COVID-19 cases across the globe during the last 13 months. The bar 13 is for January 2021 with data till January 24 , 2021 crisis for testing kits as the number of cases has started to pile up due to the virulence of the disease [9]. According to the latest guidelines, the testing method for COVID-19 is the reverse transcription polymerase chain reaction (RT-PCR). The test uses a respiratory sample that can be obtained by using a nasopharyngeal swab or sputum sample [10]. The RT-PCR test is reliable in the virulent period of the first week of infection but as time passes the virus might not appear in the throat as it completely moves down to the lungs and keeps multiplying. In this case, the coughed-up sputum samples are used to test [10].

Over the last few months, many researchers have been actively contributing towards methodological development for early COVID-19 detection and screening. This has been possible due to the recent developments of artificial intelligence (AI) and machine learning (ML)-based tools and techniques which have also been applied successfully to other tasks such as anomaly detection [12-14], biological data mining [15, 16], cyber security [17], disease detection [18-20], earthquake prediction [21], financial prediction [22], text analytics [23, 24] and urban planning [25]. Several AI and ML driven approaches have been developed to support COVID-19 [26] through analyzing lung images acquired by means of Computed Tomography (CT) [9], CXR [11][27], safeguarding workers in workplaces [28], identifying symptoms using fuzzy systems [29], and supporting hospitals using robots [30]. Many of the proposed solutions are based on computationally extensive deep learning (DL) models which are highly complex in nature and often have unreasonable computational costs.

The previously proposed DL models require extensive amounts of data in order to be trained, which could be difficult to obtain, in case of pandemic such as COVID-19. Hence, we require a robust solution that can work on small dataset and has comparable or higher accuracy than state-ofthe-art DL models. Therefore, by using the inherent property of evolutionary algorithms of managing the smaller datasets with abridged computational complexity and relatively higher accuracy, we have developed a model which can be trained with smaller datasets and still yield respectable metrics.

In this paper, we propose a pipeline that uses CXR images to detect the COVID-19. In this pipeline the features from 
the radiographs are extracted and the relevant features are then selected using a modified Social Group Optimization (SGO) algorithm which we call Hybrid SGO algorithm (HSGO). The features selected using HSGO were then used in classifying the radiographs using the support vector classifier (SVC), K-nearest neighbor (KNN), decision tree (DT), and random forest (RF).

Towards the development of an improved, reliable and accurate pipeline, the current work can be summarized through the following highlights:

1. A model is proposed to classify COVID-19 infected patients from their CXR images.

2. The model is trained and tested on open dataset of COVID-19 infected CXR images sourced from Kaggle [31-33].

3. Hybrid Social Group Optimization algorithm is proposed which is used to select features from the CXR images.

4. The selected feature set is then used to classify the CXR images using various classifiers.

5. The proposed model achieves an accuracy of $99.65 \%$ using the Support Vector Classifier.

6. Deep learning counterparts had a maximum accuracy of $99.27 \%$

7. The proposed method has a higher accuracy and significantly lower training time compared to any available state-of-the-art deep learning algorithms.

The remainder of the article is organized as follows: Section 2 depicts the related works, the proposed method is described in Section 3, Section 4 details the experimentation process and results, followed by Section 5 which concludes the work and finally Section 6 highlights the possible future developments.

\section{Related Works}

Since early 2020, several studies have been reported in the literature highlighting the shortcomings of RT-PCR testing, which includes a high false-negatives rate [34] and a short window for detection. In this public health emergency where the number of cases is increasing every day, the low sensitivity and the high rate of falsenegatives means that patients will not be identified accurately, which hampers the chance of receiving appropriate treatment. The infected people, also run the risk of infecting others, thereby compounding the threat. In [35] the authors have conducted a thorough study on the spread and fatality rate of the pandemic, showing how fatality can be greatly reduced by minimizing the exposure of vulnerable groups to COVID-19.
The Computed Tomography (CT) scan of the chest is one of the most important methods in the diagnosis of pneumonia. Ai et al. showed a strong correlation between chest CT scans and RT-PCR test results in the identification of COVID-19 [36]. Apostolopoulos and Mpesiana [37] have proposed an automated COVID19 detection system that utilizes CXR scans of the patient's chest to diagnose the disease by using transfer learning with convolutional neural network (CNN). Some researchers have also shown $\mathrm{CNN}$ to be a great tool for identification of COVID-19 from CXR radiographs [38]. In [39], authors have suggested anti-aliased convolutional networks for detection of lung diseases. Also, a number of reported work have shown that chest CT scans are an important tool in COVID-19 diagnosis [9, 40-42].

Evolutionary algorithms have always played a critical role in medical image analysis by reducing the overall computation required by intensive DL or ML algorithms. Rundo et al. [43] surveyed the literature for the state-ofart of nature-inspired medical images analysis methods focusing on bio-medical data integration. Mostafa et al. [44] used whale optimization algorithm to segment liver from MRI scans by extracting features from different segments of the image with an accuracy of $97.5 \%$. Woźniak et al. [45] used several bio-inspired algorithms to successfully detect pulmonary diseases from CXR images. The authors obtained the best accuracy of $82.22 \%$ using the particle swarm optimization algorithm. González-Patiño et al. [46] proposed a novel bio-inspired method based on bat algorithm for early identification of breast cancer by analyzing mammographic images with an accuracy of $97.42 \%$. Hemanth and Anitha [47] proposed a modified genetic algorithm to classify brain images from four different classes with a final accuracy of 98\%. Agrawal et al. [48] proposed a hybrid adaptive cuckoo search-squirrel search algorithm to analyze Brain MRI scans by obtaining optimal multi-level thresholds using maximization of the edge magnitude information. Wachs-Lopes et al. [49] discusses seven recent bio-inspired algorithms over multi-thresholding segmentation of medical images. The algorithms were tested for a range of values of non-extensivity parameter ('q'), which is an essential parameter for Tsallis entropy. The firefly algorithm had the best performance and the Grey Wolf Optimizer with the fastest convergence.

There have been a number of studies on COVID19 using CXR images, Ozturk et al. [50] proposed DarkCOVIDNet using CNN and DarkNet, 2-D convolution and Max Pooling for identification of COVID-19 from CXR images. The accuracy achieved by this model was 98.08\%. Toğaçar et al. [51] used social mimic optimization along with SqueezeNet and MobileNetV2 deep learning 
architectures for the identification of COVID-19 from CXR images. The accuracy of this model was 99.27 (binary class) for covid-19 images, but the overall accuracy was $98.3 \%$ (COVID and Normal CXR images, multi-class). The accuracy of this model was indeed good, however, the method was not computationally efficient with two deep learning models. Panwar et al. [52] presented a model for fast identification of COVID19 using the Vgg-16 and CNN deep learning-based model. The accuracy for the binary class for classifying COVID19 images was $97.97 \%$ and normal image detection accuracy was $98.68 \%$. Pereira et al. [53] used the pretrained CNN network, the F1-score achieved 0.89, which is quite low as acknowledged by the author as well. Waheed et al. [54] proposed COVIDGAN an auxiliary classifier generative adversarial network (GAN), the method had a limitation, that very small dataset was used in order to train, then GAN was used to create synthetic dataset. The method achieved an accuracy between 85\%-95\% despite being biased towards the generated image dataset. Abdel-Basset et al. [55] proposed an improved marine predators algorithm, bettering the results obtained by other bio-inspired algorithm for COVID-19 detection with an overall fitness function value obtained 66.26. Oh et al. [56] proposed a method to segmentation of the lung images along with FC-DenseNet103 gave an overall accuracy of $88.9 \%$ for classification of COVID-19 images and 95\% accuracy on classifying COVID-19 and Normal images. Vaid et al. [57] used simple pre-trained deep learning models and achieved an accuracy of $96.3 \%$ in classifying COVID-19 images. Mahmud et al. [58] proposed a multi-dilated CNN and achieved an accuracy of $97.4 \%$ for successfully detecting COVID-19 images. Moreover, Dey et al. [9] proposed SGO assisted Kapoor's entropy to segment COVID-19 specific regions in CT scan images and used K-nearest neighbor method to classify. The method achieved an accuracy of $87.75 \%$.

\section{Proposed Method}

This work proposes a computer-aided diagnosis system that can automatically detect COVID-19 by using CXR radiographs of the patients. This system is expected to contribute in aiding doctors in the decision process, thereby reducing the time taken for accurate diagnosis and thus, hopefully, reducing the overall pressure on the medical staff as well. However, this should be noted that the has not been clinically validated like many other methods proposed in the literature and should not be used as an independent means to diagnose COVID-19.

The proposed pipeline consists of the following steps:

1. Preprocessing of the lung CXR radiographs;

2. Feature extraction from CXR radiographs;

3. Selection of relevant features using the HSGO algorithm;

4. Classification of the extracted features from the radiographs as healthy or infected.

The aforementioned pipeline is depicted in Fig. 3.

\section{Preprocessing and Feature Extraction}

To detect COVID-19 infection through an automated classification method, features need to be extracted from the CXR images. In the first step, the given radiographs were resized to $100 \times 100$ from their original size of $1024 \times 1024$, which boosted computational speeds significantly and also reduced the extracted feature pool
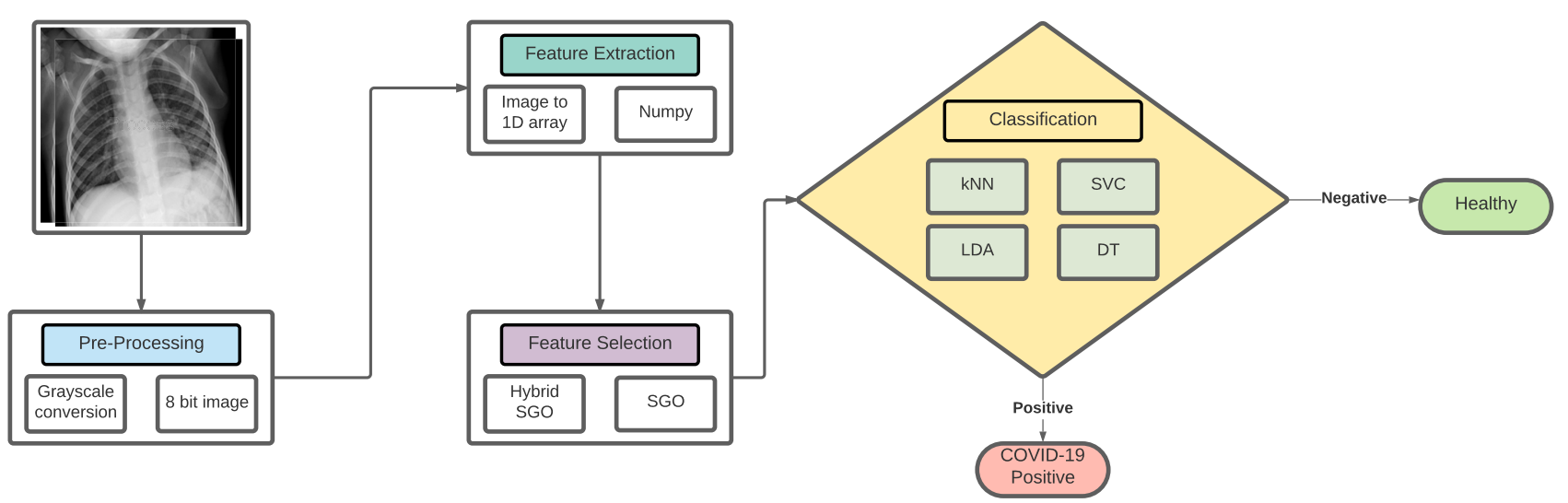

Fig. 3 Flowchart of the proposed pipeline. It shows the main steps as: preprocessing, feature extraction, feature selection, and classification 
Fig. 4 Preprocessing and feature extraction of lung CXR images

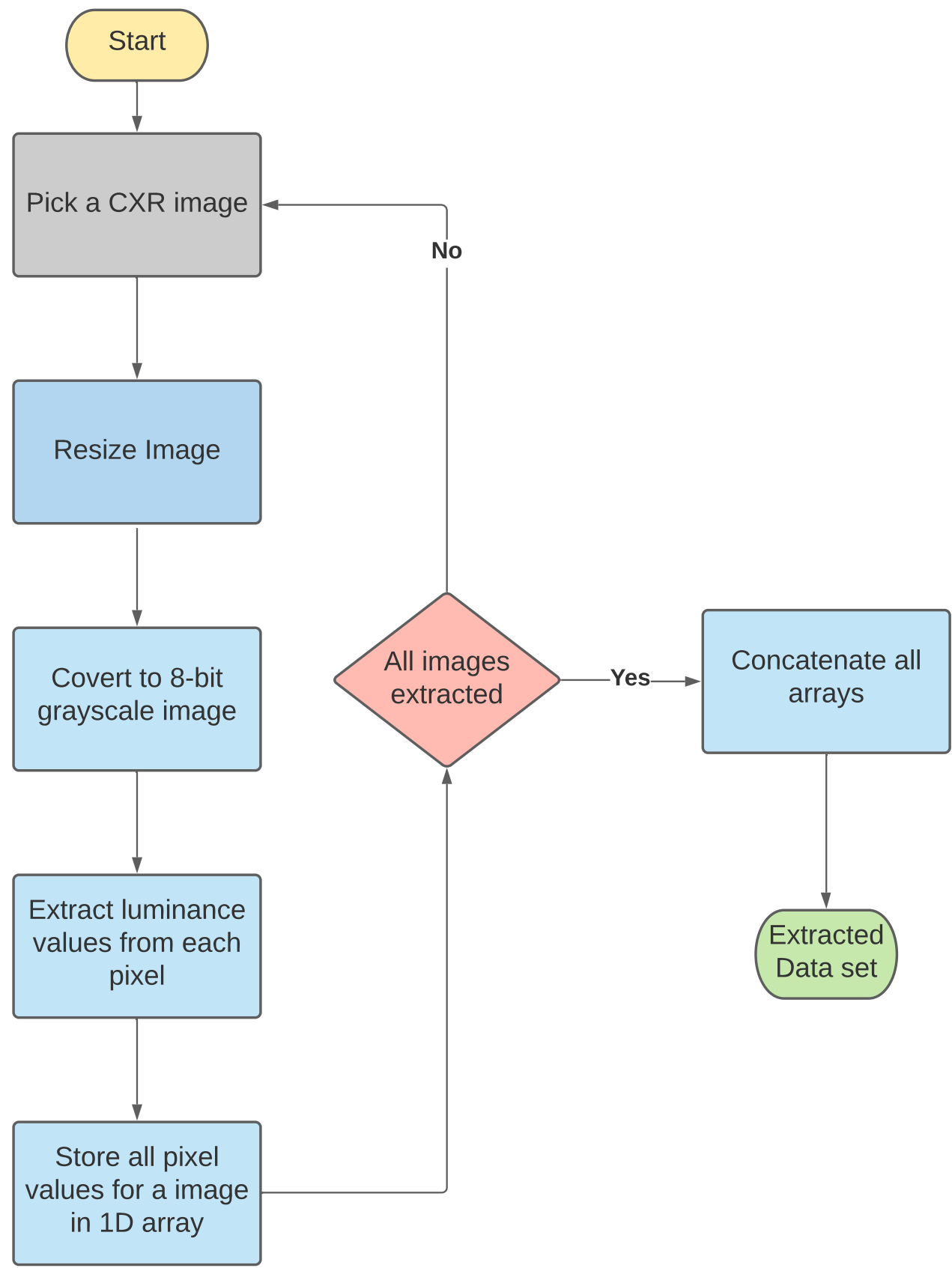

without compromising accuracy. All the features were extracted using Pillow 7.1.2 in grayscale mode [59] which extracts every pixel in the image by assigning a value according to the luminosity level of the pixel. The value is determined by the ITU-R 601-2 luma transform for every pixel in image. The lung CXR is first converted to a 8-bit image where every bit stores black and white levels thereby allowing 256 different shades. By doing this we can keep the subtle details and also minimize the extracted feature pool. Figure 4 depicts the preprocessing and extraction process in detail.

Other extraction methods like bi-level mode that converts a lung CXR to 1-bit image where value for every pixel is determined using Floyd-Steinberg dither to approximate luminosity levels, Palette mode which converts and gives 8 bit of data for each pixel allowing 256 colors and RGB mode that converts and stores the true color value of every pixel using $3 \times 8$ bits were tested. All the aforementioned methods performed worse than 
the grayscale method. In case of Bi-level mode the final accuracy was considerably lower as many details required to classify an image were lost in conversion, whereas Palette and RGB mode both preserved the details but the number of features extracted was excessive for the problem at hand. Thus, using Grayscale mode the lung CXRs were converted and a total of 10,000 features were extracted from each one. The extracted feature pool was then subjected to feature selection, as described in subsequent sections.

\section{Feature Selection}

In this section, we discuss feature selection from the extracted dataset using the proposed HSGO algorithm. This step is essential for reducing computational costs and also boosts accuracy. The feature selection method is based on the SGO algorithm, which is described below.

\section{Social Group Optimization (SGO)}

The SGO algorithm, proposed by Satapathy and Naik [60], is a meta-heuristic model based on population behavior that is inspired by the human group's ability to solve a complex problem. It is based on the observation that a group's problem-solving capability is better than an individual's problemsolving ability as it exploits every member's unique traits to solve a complex problem. Every person in the population is a candidate solution that has some knowledge about the solution to the given problem. The person with the best solution in the population imparts its knowledge to others, thereby increasing the overall knowledge of the entire population.

Mathematically, the SGO algorithm can be expressed as: let $N$ be the number of persons in the population and each person is defined as $P_{i}=\left(x_{1}, x_{2}, x_{3}, \cdots, x_{D}\right)$ where $D$ is the number of features which uniquely define a person. The features of every person need to be optimized to yield the best solution to the problem. For a given problem, the fitness function can be defined as $f_{i}$ where $0<i<N$ for every person in the population.

Improving Phase Every person acquires some knowledge from the best person by the following function: $x_{i, j}($ new $)=c \times x_{i, j}($ old $)+r \times\left(\right.$ gbest $_{j}-x_{i, j}($ old $\left.)\right)$ here, gbest $_{j}$ is the population's best person, i.e., person with the best fitness value, $c$ is the self-introspection factor, $r$ is any random value $0<r<1$, and $x_{i, j}$ (new) is the $j$ th feature for the $i$ th person in the population which is accepted if it gives better fitness value.

Acquiring Phase Every person in the population learns from random persons in the population and from the population's best person by the following function: $x_{i, j}($ new $)=x_{i, j}($ old $)+r 1 \times\left(x_{r, j}-x_{i, j}\right)+r 2 \times\left(\right.$ gbest $\left._{j}-x_{i, j}\right)$ where $x_{i, j}($ old $)$ is the initial value of the $j$ th feature for the $i$ th person in the population, gbest $_{j}$ is the population's best person, i.e., person with the best fitness value, $c$ is the self-introspection factor, $r 1$ and $r 2$ are any random values $0<r 1, r 2<1$, and $x_{i, j}(n e w)$ is the $j$ th feature for the $i$ th person in the population which is accepted if it gives better fitness value.

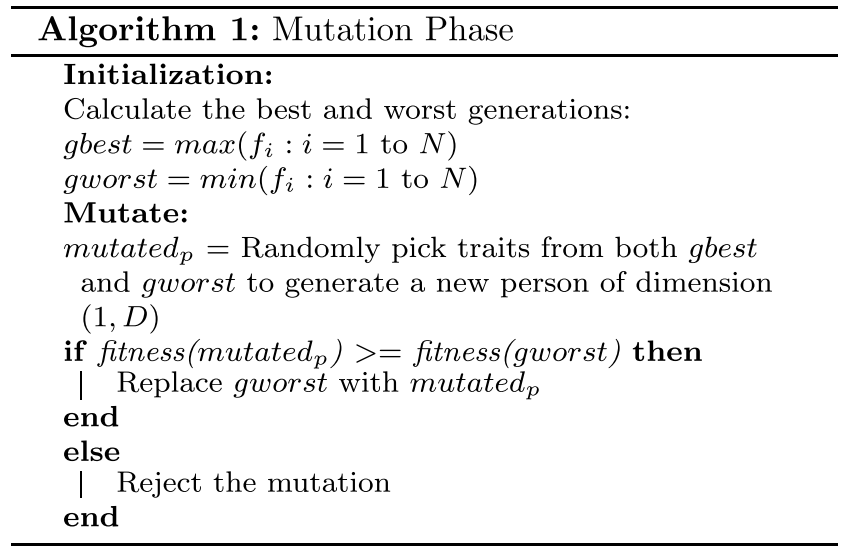

\section{Hybrid Social Group Optimization (HSGO)}

The HSGO algorithm is an improved version of the SGO algorithm, which has been developed to select optimal features from a feature pool. By following a wrapper-based approach, HSGO gives the optimal feature set in considerably fewer iterations. Theoretically, both SGO and HSGO should eventually give the optimal feature set but in our experiments HSGO outperforms SGO and other conventional feature selection methods by selecting a markedly smaller feature set which also gives a noticeably better accuracy. Table 1 compares HSGO with SGO and other conventional methods. This improvement can be attributed to addition of a random factor which helps HSGO to overcome local minima or maxima traps, when compared to the traditional SGO algorithm. The SGO algorithm when used for feature selection, tends to yield the same results after a few generations of notable improvement in the population. The effect of this random factor is evident especially when the number of features in the dataset are high ( $>1000)$. The random factor is obtained by introducing a new step called the Mutation phase, this phase is explained in Algorithm 1. Algorithm 2 shows the proposed HSGO algorithm which can be broadly divided to the following steps.

Table 1 Comparison of Feature Selection Methods

\begin{tabular}{llll}
\hline Selection Method & $\begin{array}{l}\text { Features } \\
\text { Selected }\end{array}$ & Accuracy (\%) & Classifier Used \\
\hline Hybrid SGO & 116 & 99.65 & SVC \\
SGO & 254 & 99.31 & SVC \\
KPCA & 500 & 99.31 & SVC \\
PCA & 511 & 99.31 & SVC \\
\hline
\end{tabular}




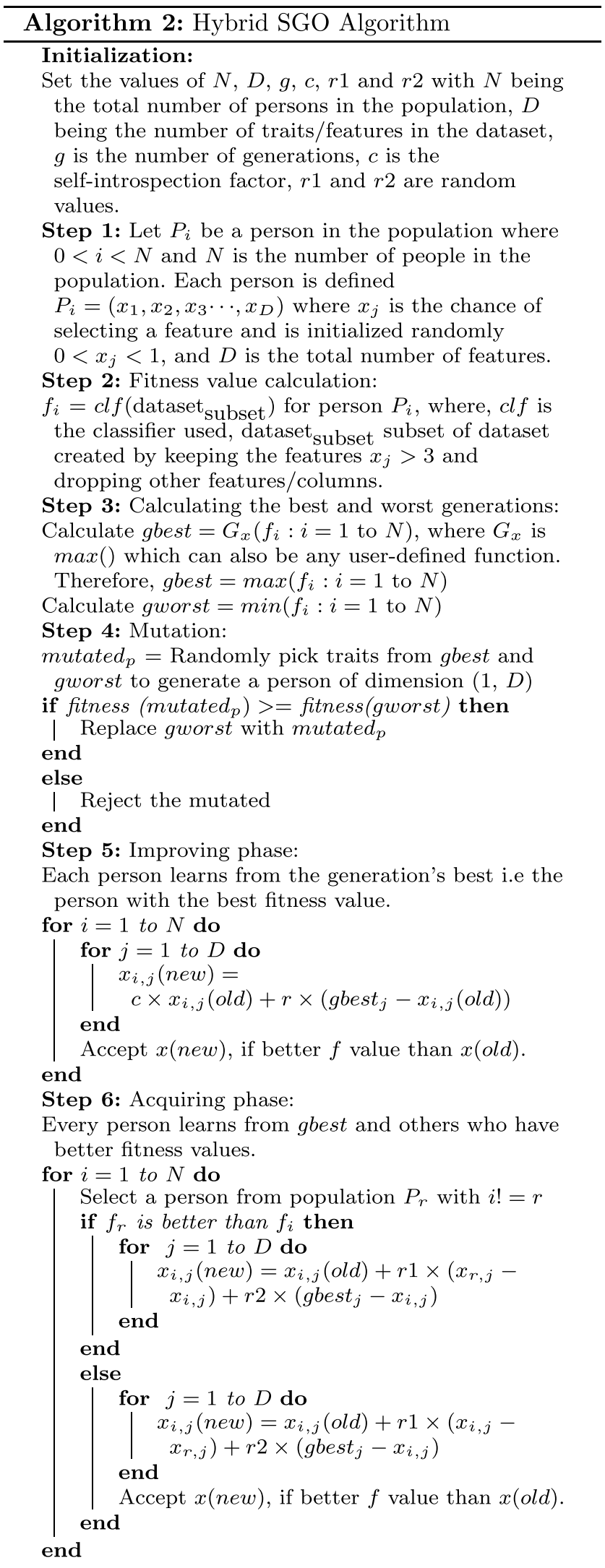

Initialization function The initialization function initializes each person in the population by making a random array of integer values ranging from 0 to 9 with dimensions $(N, D)$ where $N$ is the number of people in population and $D$ is total extracted features. The range of 0 to 9 was chosen to allow enough randomness in features of every person in the population while at the same time making it much easier to trace the learning steps. The initialization and improvement process is shown pictorially in Fig. 5.

Fitness function The fitness function used here is the classifier which will be used on the optimal subset of features. It gives the fitness and the accuracy of the current subset of the dataset by assigning fitness values to each person in the population after every generation.

$f_{i}=\operatorname{classifier}\left(P_{i}\right)$

where, classifier is the classifier being used, $P_{i}$ is the $i$ th person in the population, and $f_{i}$ is the calculated fitness.

Mutation Phase The purpose of the mutation phase is to facilitate continuous improvement in the population by constantly introducing a new person whose traits are notably different from the persons currently in the population. Algorithm 1 shows the mutation phase of the process.

A subset of the dataset is created for each person in population, every generation, by selecting only the corresponding columns of the dataset where the persons feature value is greater than 3 . Initially, we selected the columns from dataset which had corresponding feature value of 5 or more, but it was changed to 3 to get a wide variety of feature subsets in the initial population. The subset created is then used to calculate accuracy using appropriate classifiers and is gradually improved with every generation to give the final selection of features that give the best accuracy and minimum computational cost.

The HSGO algorithm selected 116 features out of 10,000 raw features extracted from lung CXR images. In other words, the algorithm selected only $1.16 \%$ features from the extracted feature set, therefore, filtering out $98.84 \%$ of insignificant features. Table 1 shows the comparison of various feature selection methods used, accuracy obtained using the feature subset and the classifier used.

\section{Experimentation and Results}

In this section, we discuss the implementation of the HSGO algorithm. It comprises of setup specifications along with the various parameters used in the algorithm and classifiers.

\section{Dataset}

The dataset used in this work was obtained from the Kaggle repository "COVID-19 Radiography Database". The database from this repository consisted of 219 
Fig. 5 Initialization and generational improvement provided by the hybrid SGO algorithm

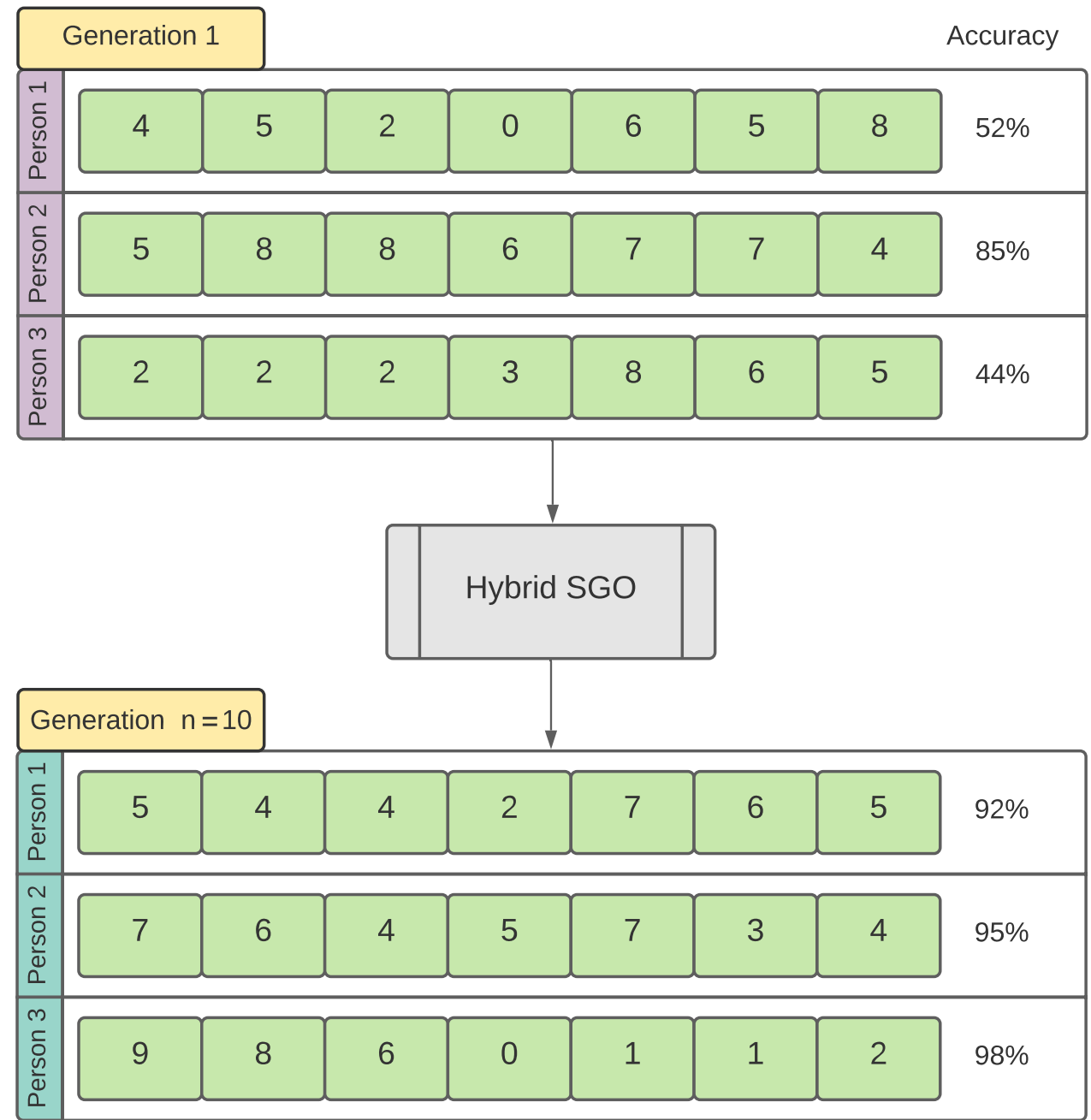

COVID-19 positive images, 1341 normal images, and 1345 viral pneumonia images. [31-33]. Adding 152 more COVID-19 positive images from other similar sources, the total number of images for this class was increased to 371. Our experiments were performed using CXR radiographs of positive COVID-19 cases and normal images from the dataset. All the images were resized from $1024 \times 1024$ to $100 \times 100$ pixels, which substantially reduces the feature pool extracted and boosts computational speed. All the results quoted in the work were achieved using the original dataset. Representative radiographs for both COVID-19 infected patients and normal cases are given in Fig. 6, respectively. As from the individual image numbers from different classes it can be seen that the dataset is imbalanced, we created 3 subsets from the full dataset for evaluation. The split ratio between the COVID-19 and non-COVID-19 CXR images were changed by either increasing or decreasing the non-COVID-19 CXR images to make the subsets to contain 30\% COVID-19-70\% non-COVID-19 images, 50\% COVID-19-50\% non-COVID-19 images, and the full dataset containing all COVID-19 and non-COVID-19 CXR images. It should be noted here that, this split was solely for the sake of evaluation, the final model was trained on the original dataset.

\section{Experimental Setup}

All tests were performed on Google Colaboratory, allowing execution of python code in browser, on Google Cloud Servers running on a Intel(R) Xeon(R) CPU @ $2.30 \mathrm{GHz}$, having 20 physical cores paired with a K80 GPU and RAM capacity up to $36 \mathrm{~GB}$. A maximum of $108 \mathrm{~GB}$ of disk space was available, out of which $33 \mathrm{~GB}$ was used for the experiments. Pillow library, a fork of Python Imaging Library was used for extracting features from the lung CXRs. All the algorithms were developed using Python 3.6.7 as the language and NumPy, Pandas, and Scikit-learn libraries. 


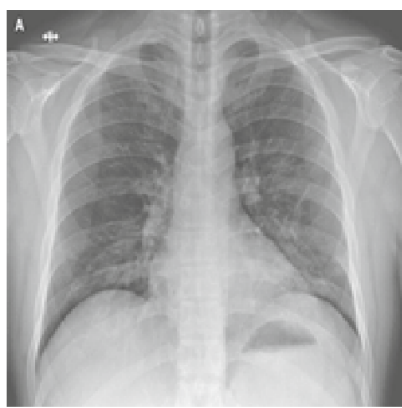

COVID-19

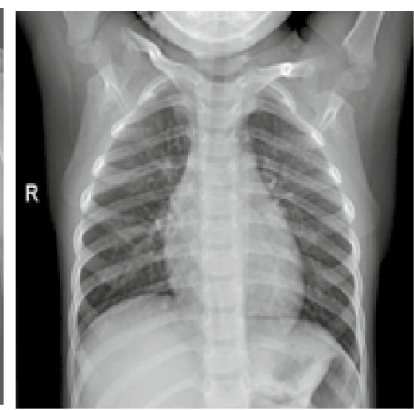

Normal
Fig. 6 Example chest X-ray images from the dateset - COVID-19 (left), and normal (right)

\section{Input Parameters}

The parameters used in the HSGO algorithm are shown in Table 2 and the parameters of the various classifiers are reported in Table 3.

Among the parameters of the HSGO algorithm, the $N$ and $D$ are constant values which are chosen as per the problem requirements. The self-introspection factor $c$ used in the improving phase controls the knowledge gained from the generation's best at each iteration and can be set from $0<c<1$. If the value of $\mathrm{c}$ is too low, the learning steps are too flat, which can be beneficial in some cases whereas higher values of $c(>0.8)$ results in steep learning steps and is generally unstable. In our experiments $c=0.7$ was used which yields a respectable learning rate without compromising stability. $r 1$ and $r 2$ are independent random numbers which affect the stochastic nature of the algorithm. The value of $r 1$ and $r 2$ can be $0<r 1, r 2<1$. We have used $r 1=0.6$ and $r 2=0.8$ in our experiments to achieve the stated results. All the parameters used in classifiers were obtained by the process of hyper parameter tuning using Grid Search algorithm. The algorithm determines the best parameters by methodically checking the specified classifier against a set of possible parameters thereby ensuring best metrics.

\section{Performance}

The proposed pipeline with HSGO algorithm was applied on the complete dataset (i.e., 371 COVID-19 positive

Table 2 Parameters for the HSGO Algorithm

\begin{tabular}{lll}
\hline Parameter & Value & Description \\
\hline$N$ & 5 & No. of Persons \\
$D$ & 10,000 & No. of features \\
$r 1$ & 0.6 & Random value $\mathrm{r} 1$ \\
$r 2$ & 0.8 & Random value $\mathrm{r} 2$ \\
$c$ & 0.7 & Self-Introspection factor \\
\hline
\end{tabular}

Table 3 Parameters for Machine Learning Algorithms

\begin{tabular}{ll}
\hline Classifier & Parameters Used \\
\hline KNN & n-neighbors $=3$, metric $=$ manhattan \\
DT & criterion $=$ gini, splitter $=$ best \\
RF & criterion $=$ gini, max-depth $=2$, n-estimators $=100$ \\
L-SVC & penalty $=$ '12', loss $=$ 'squaredhinge', maxiter $=1000$ \\
SVC & C $=1.0$, kernel $=$ 'rbf', degree $=3$, gamma = 'scale', \\
& maxiter $=-1$ \\
PCA & n_components $=0.98$, random_state $=$ None \\
KPCA & kernel $=$ 'linear', n_components $=500$
\end{tabular}

CXR images and 1341 non-COVID-19 CXR images) and the classification of the features was performed by five different methods, namely, KNN, DT, RF, L-SVC, and SVC. As shown in Fig. 7, the best accuracy of $99.65 \%$ was obtained with the SVC classifier and this was consistent with the F1-score obtained for the SVC method reported in Table 4.

To assess the effect of the number of COVID-19 images on the accuracy, the proposed pipeline with HSGO algorithm and SVC classifier was applied on three different subsets of data (see "Dataset" section for details). It was observed that the highest accuracy of $99.65 \%$ was obtained when the complete dataset was used. Also, comparing with other optimization algorithms, as shown in Fig. 8, this highest accuracy was obtained with the HSGO algorithm on the complete dataset in comparison to other methods (such as SGO, KPCA, and PCA) and dataset size. On the same dataset the SGO, KPCA, and PCA methods provided an accuracy

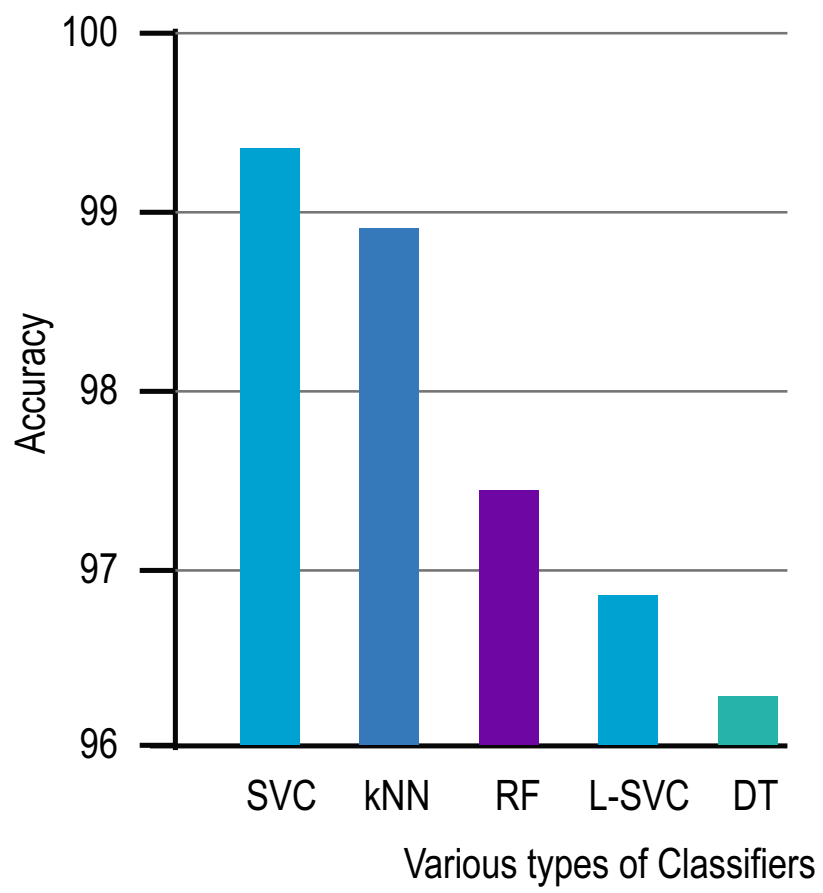

Fig. 7 Classification accuracy of various classifiers 
Fig. 8 Effect of number of images from COVID-19 and non-COVID-19 classes on classification performance

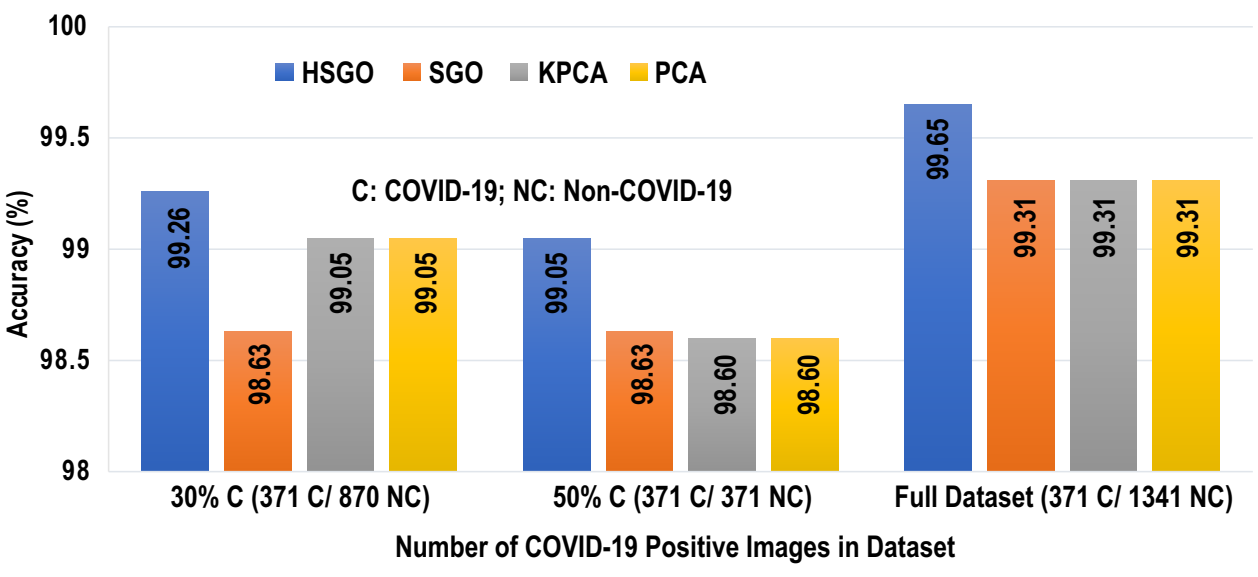

of $99.31 \%$. When the number of COVID-19 positive CXR images were $30 \%$ and $50 \%$ and the non-COVID-19 images were $70 \%$ and $50 \%$, respectively, the proposed HSGO algorithm outperformed all other methods. In case of the 3:7 dataset, the highest accuracy was $99.05 \%$ with HSGO and in case of the 1:1 dataset, the highest accuracy was $99.26 \%$.

Evidently, the performance of the pipeline is consistent even after altering the composition of the dataset showing the classification accuracy of minority class, i.e., COVID-19 is maintained. Based on the above discussions, it can be claimed that the proposed HSGO-based pipeline, in combination with the SVC, is capable of detecting COVID-19 from CXR radiographs remarkably well.

\section{Benchmarking with State-of-the-Art Deep Learning Models and Evolutionary Algorithms}

The results obtained with the HGSO algorithm were also compared with other similar bio-inspired algorithms namely Chaotic Crow Search Optimization algorithm (CSO) and Spider-Monkey Optimization algorithm (OSMO). These algorithms are tweaked versions of the original crow search algorithm and spider monkey optimization algorithm, respectively. The CSO algorithm

Table 4 Performance comparison of different classifiers

\begin{tabular}{llll}
\hline Class. & Prec. & Sens. & F1S \\
\hline KNN & 0.9896 & 0.9897 & 0.9897 \\
DT & 0.9489 & 0.9591 & 0.9611 \\
RF & 0.9758 & 0.9761 & 0.9759 \\
L-SVC & 0.993 & 0.993 & 0.993 \\
SVC & 0.9966 & 0.9965 & 0.9965 \\
\hline
\end{tabular}

Class Classifier, Prec Precision, Sens Sensitivity, F1S F1 score is a meta-heuristic optimizer which takes inspiration from a crow's searching methods to hide extra food and its retrieval when needed [61]. On the other hand, the OSMO algorithm is a swarm intelligence technique [62] that relies on the collective intelligence of a group to solve the problem at hand. Both algorithms were used as a feature selection method for the same dataset and the selected feature set was then used with every classifier. The maximum accuracy obtained were saved and plotted. As seen in Fig. 9, the proposed method performs better than aforementioned meta-heuristics.

The authors in [38] used four pre-trained deep CNN architectures, namely, AlexNet, ResNet18, SqueezeNet and DenseNet201 to classify normal and COVID-19 infected radiographs with an accuracy as high as $98.3 \%$ using the SqueezeNet. A comparison of the accuracy of our model against the state-of-the-art DL (as discussed in section 2) and other optimized algorithms have been depicted in the bar graph in Fig. 9, Evidently the proposed method outperforms every other method with $99.65 \%$ accuracy using the SVC classifier.

\section{Conclusion}

The ongoing global pandemic due to the COVID-19 outbreak has led to a global crisis worldwide. Even developed countries are struggling to cope with the demand for medical supplies and testing kits. The shortage of testing kits especially hampers efforts to stop the spread of the disease, as many cases go undetected, which may lead to even more infections of COVID-19. Early diagnosis of the disease is essential to stop further spread and reduce mortality rates. The proposed solution of a computer-aided diagnosis system, uses CXR radiographs of patients to automatically predict COVID-19. Our experimental results show that the model with SVC yields the highest accuracy 
Fig. 9 Accuracy comparison with other Deep Learning and other Bio-Inspired Algorithms. References: nCOVnet [52], SqueezeNet [38], Squeeze\&MobileNetV2 [51], DarkCOVIDNet [50], Alexnet [38], COVxnet [58], ResNet [38], DL-VGG-19 [57], COVIDGAN [54], FC-DenseNet103 [56], OSMO [61], and CSO [62]

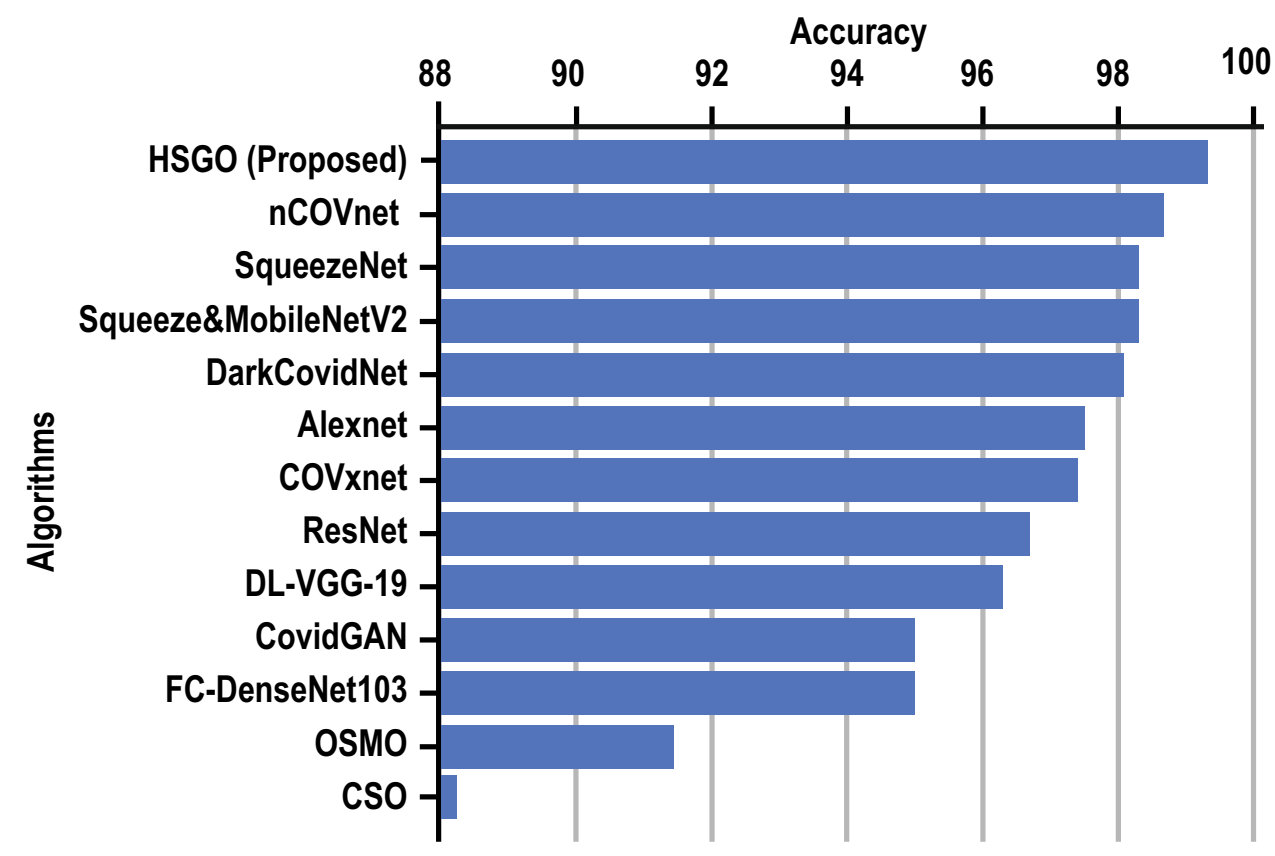

of $99.65 \%$ among all classifiers. The proposed pipeline, due to its high accuracy and precision, can be used to develop mobile applications that can be an aid in early diagnosis of COVID-19 for medical practitioners.

\section{Future Scope}

The proposed pipeline's accuracy can be further improved. For instance, the HSGO model can be made more accurate by increasing the number of COVID-19 chest CXR images. The effects of numerous parameters used in HSGO can be studied in depth on different datasets to unravel how they all come together and affect the final results. Alternative feature extraction methods can be experimented with the proposed pipeline to further enhance the end result. The Mutation phase of the HSGO can also be adjusted according to the problem at hand, thereby allowing widespread applications in countless real-world problems.

Author Contributions This work was carried out in close collaboration between all co-authors. All authors have contributed to, seen and approved the final manuscript.

Code Availability The code can be accessed via the following GitHub repository: https://github.com/Enixes/Hybrid-Social-GroupOptimization-algorithm.

\section{Declarations}

Ethical Approval All procedures performed in studies involving human participants were in accordance with the ethical standards of the institutional and/or national research committee and with the 1964 Helsinki declaration and its later amendments or comparable ethical standards.

Informed Consent Informed consent was obtained from all individual participants included in the study.

Conflict of Interest The authors declare that the research was conducted in the absence of any commercial or financial relationships that could be construed as a potential conflict of interest.

Open Access This article is licensed under a Creative Commons Attribution 4.0 International License, which permits use, sharing, adaptation, distribution and reproduction in any medium or format, as long as you give appropriate credit to the original author(s) and the source, provide a link to the Creative Commons licence, and indicate if changes were made. The images or other third party material in this article are included in the article's Creative Commons licence, unless indicated otherwise in a credit line to the material. If material is not included in the article's Creative Commons licence and your intended use is not permitted by statutory regulation or exceeds the permitted use, you will need to obtain permission directly from the copyright holder. To view a copy of this licence, visit http://creativecommons.org/licenses/by/4.0/.

\section{References}

1. Zhu N, et al. A Novel Coronavirus from Patients with Pneumonia in China: 2019. N Engl J Med. 2020;382(1):727-33.

2. Hui DS, et al. The continuing 2019-nCoV epidemic threat of novel coronaviruses to global health -The latest 2019 novel coronavirus outbreak in Wuhan. China. Int J Infect Dis. 2020;91(1):264-6.

3. Fraire AE, Woda BA, Welsh RM, Kradin RL. Viruses and the Lung. Berlin Heidelberg: Springer-Verlag, Berlin Heidelberg; 2014.

4. CDC. SARS Basics Fact Sheet;. Available at https://www.cdc.gov/ sars/about/fs-sars.html (2020/05/20). 
5. Annelies WS, CJ C, VJ L. Can we contain the COVID-19 outbreak with the same measures as for SARS? Lancet Infect Disease. 2020;20(5):e102-107

6. Shereen MA, Khan S, Kazmi A, Bashir N, Siddique R. COVID19 infection: Origin, transmission, and characteristics of human coronaviruses. J Adv Res. 2020;24:91-8.

7. Rothan HA, Byrareddy SN. The epidemiology and pathogenesis of coronavirus disease (COVID-19) outbreak. J Autoimmun. 2020;109:102433.

8. Sohrabi C, et al. World Health Organization declares global emergency: A review of the 2019 novel coronavirus (COVID19). Int J Surg. 2020;76:71-6.

9. Dey N, Rajinikanth V, Fong SJ, Kaiser MS, Mahmud M. Social-group-optimization assisted Kapur's Entropy and Morphological Segmentation for automated detection of COVID19 infection from computed tomography images. Cogn Comput. 2020;12(5):1011-23.

10. CDC. Information for Laboratories;. Available at https://www. cdc.gov/coronavirus/2019-ncov/lab/index.html (2020/05/20).

11. Rousan LA, Elobeid E, Karrar M, et al. Chest X-ray findings and temporal lung changes in patients with COVID-19 pneumonia. BMC Pulm Med. 2020;245:

12. Yahaya SW, Lotfi A, Mahmud M. A consensus novelty detection ensemble approach for anomaly detection in activities of daily living. Appl Soft Comput. 2019;83:105613.

13. Fabietti M, Mahmud M, Lotfi A, Averna A, Guggenmo D, Nudo R, et al. Neural Network-based Artifact Detection in Local Field Potentials Recorded from Chronically Implanted Neural Probes. In: Proc. IJCNN; 2020. p. 1-8.

14. Ali HM, Kaiser MS, Mahmud M. Application of Convolutional Neural Network in Segmenting Brain Regions from MRI Data. In: Goel V, Shan C, editors. Liang P. Brain Informatics. Lecture Notes in Computer Science. Cham: Springer International Publishing; 2019. p. 136-146.

15. Mahmud M, Kaiser MS, Hussain A, Vassanelli S. Applications of deep learning and reinforcement learning to biological data. IEEE Trans Neural Netw Learn Syst. 2018;29(6):2063-79.

16. Mahmud M, Kaiser MS, McGinnity TM, Hussain A. Deep Learning in Mining Biological Data. Cogn Comput. 2021;13(1):1-33.

17. Mahmud M, Kaiser MS, Rahman MM, Rahman MA, Shabut A, Al-Mamun S, et al. A brain-inspired trust management model to assure security in a cloud based IoT framework for neuroscience applications. Cogn Comput. 2018;10(5):864-73.

18. Noor MBT, Zenia NZ, Kaiser MS, Mahmud M, Al Mamun S. Detecting Neurodegenerative Disease from MRI: A Brief Review on a Deep Learning Perspective. In: Goel V, Shan C, editors. Liang P. Brain Informatics. Lecture Notes in Computer Science. Cham: Springer International Publishing; 2019. p. $115-125$

19. Noor MBT, Zenia NZ, Kaiser MS, Al Mamun S, Mahmud M. Application of deep learning in detecting neurological disorders from magnetic resonance images: a survey on the detection of Alzheimer's disease. Parkinson's disease and schizophrenia. Brain informatics. 2020;7(1):1-21.

20. Miah Y, Prima CNE, Seema SJ, Mahmud M, Kaiser MS. Performance comparison of machine learning techniques in identifying dementia from open access clinical datasets. In: Proc. ICACIn. Springer, Singapore; 2021. p. 79-89.

21. Al Banna MH, Taher KA, Kaiser MS, Mahmud M, Rahman MS, Hosen AS, et al. Application of artificial intelligence in predicting earthquakes: state-of-the-art and future challenges. IEEE Access. 2020;8:192880-923.

22. Orojo O, Tepper J, McGinnity TM, Mahmud M. A Multirecurrent Network for Crude Oil Price Prediction. In: Proc. SSCI; 2019. p. 2940-2945.
23. Watkins J, Fabietti M, Mahmud M. SENSE: a Student Performance Quantifier using Sentiment Analysis. In: Proc. IJCNN; 2020. p. 1-6.

24. Rabby G, Azad S, Mahmud M, Zamli KZ, Rahman MM. TeKET: a tree-based unsupervised keyphrase extraction technique. Cogn Comput. 2020;12(4):811-33.

25. Kaiser MS, Lwin KT, Mahmud M, Hajializadeh D, Chaipimonplin T, Sarhan A, et al. Advances in crowd analysis for urban applications through urban event detection. IEEE Trans Intell Transp Syst. 2018;19(10):3092-112.

26. Mahmud M, Kaiser MS. Machine Learning in Fighting Pandemics: A COVID-19 Case Study. In: Santosh KC, Joshi A, editors. COVID-19: Prediction, Decision-Making, and its Impacts. Lecture Notes on Data Engineering and Communications Technologies. Singapore: Springer; 2021. p. 77-81.

27. Aradhya VNM, Mahmud M, Agarwal B, Kaiser MS. One Shot Cluster based Approach for the Detection of COVID-19 from Chest X-Ray Images. Cogn Comput. 2021;p. 1-9. [Online First, doi: https://doi.org/10.1007/s12559-020-09774-w].

28. Kaiser MS, et al. iWorkSafe: Towards Healthy Workplaces during COVID-19 with an Intelligent pHealth App for Industrial Settings. IEEE Access. 2021;9:13814-13828 https://doi. org/10.1109/ACCESS.2021.3050193.

29. Bhapkar HR, Mahalle PN, Shinde GR, Mahmud M. Rough Sets in COVID-19 to Predict Symptomatic Cases. In: Santosh KC, Joshi A, editors. COVID-19: Prediction, Decision-Making, and its Impacts. Lecture Notes on Data Engineering and Communications Technologies. Singapore: Springer; 2021. p. $57-68$.

30. Kaiser MS, Al Mamun S, Mahmud M, Tania MH. Healthcare Robots to Combat COVID-19. In: Santosh KC, Joshi A, editors. COVID-19: Prediction, Decision-Making, and its Impacts. Lecture Notes on Data Engineering and Communications Technologies. Singapore: Springer; 2021. p. 83-97.

31. SIRMI. COVID-19 Database. Societa Italiana di Radiologia;. Available at https://www.sirm.org/category/senza-categoria/ covid-19/ (2020/05/20).

32. Cohen JP, Morrison P, Dao L. COVID-19 image data collection. GitHub; 2020. Accessed on 25/07/2020. Available from: https:// github.com/ieee8023/covid-chestxray-dataset.

33. Mooney P. Chest X-Ray Images (Pneumonia);. Available at https://www.kaggle.com/paultimothymooney/chest-xraypneumonia $(2020 / 05 / 20)$.

34. Prinzi A. False Negatives and Reinfections: the Challenges of SARS-CoV-2 RT-PCR Testing;. Available at https://asm.org/ Articles/2020/April/False-Negatives-and-Reinfections-theChallenges-of (2020/05/20).

35. Anderez DO, Kanjo E, Pogrebna G, Kaiwartya O, Johnson SD, Hunt JA. A COVID-19-Based Modified Epidemiological Model and Technological Approaches to Help Vulnerable Individuals Emerge from the Lockdown in the UK. Sensors (Basel). 2020;20:20(17):4967.

36. Ai T, et al. Correlation of Chest CT and RT-PCR Testing in Coronavirus Disease 2019 (COVID-19) in China: A Report of 1014 Cases. Radiology. 2020;296(2):E32-E40.

37. Apostolopoulos ID, Mpesiana TA. Covid-19: automatic detection from X-ray images utilizing transfer learning with convolutional neural network. Phys Eng Sci Med. 2020;76:71-6.

38. Chowdhury ME, et al. Can AI help in screening Viral and COVID19 pneumonia? CoRR. 2020;abs/2003.13145:1-12.

39. Singh J, Tripathy A, Garg P, Kumar A. Lung tuberculosis detection using anti-aliased convolutional networks. Procedia Comput Sci. 2020;173:281-90.

40. Tenda ED, et al. The Importance of Chest CT Scan in COVID-19: A Case Series. Acta Med Indones. 2020;68-73. 
41. Yang R, et al. Chest CT Severity Score: An Imaging Tool for Assessing Severe COVID-19. Radiol: Cardiothorac Imaging. 2020;2(2):1-23.

42. Caruso D, et al. Chest CT Features of COVID-19 in Rome. Italy. Radiology. 2020;296(2):E79-E85.

43. Rundo L, Militello C, Vitabile S, Russo G, Sala E, Gilardi MC. A survey on nature-inspired medical image analysis: a step further in biomedical data integration. Fundam Inform. 2020;171(1-4):345-65.

44. Mostafa A, Hassanien AE, Houseni M, Hefny H. Liver segmentation in MRI images based on whale optimization algorithm. Multimed Tools Appl. 2017;76(23):24931-54.

45. Woźniak M, Połap D. Bio-inspired methods modeled for respiratory disease detection from medical images. Swarm Evol Comput. 2018;41:69-966.

46. González-Patiño D, Villuendas-Rey Y, Argüelles-Cruz AJ, Karray F. A novel bio-inspired method for early diagnosis of breast cancer through mammographic image analysis. Appl Sci. 2019;9(21):4492.

47. Hemanth DJ, Anitha J. Modified Genetic Algorithm approaches for classification of abnormal Magnetic Resonance Brain tumour images. Appl Soft Comput. 2019;75:21-8.

48. Agrawal S, Samantaray L, Panda R, Dora L. A New Hybrid Adaptive Cuckoo Search-Squirrel Search Algorithm for Brain MR Image Analysis. In: Studies in Computational Intelligence. Springer Singapore; 2019. p. 85-117.

49. Wachs-Lopes G, Santos R, Saito N, Rodrigues P. Recent natureInspired algorithms for medical image segmentation based on tsallis statistics. Commun Nonlinear Sci Numer Simul. 2020;88:105256.

50. Ozturk T, et al. Automated detection of COVID-19 cases using deep neural networks with X-ray images. Comput Biol Med. 2020;121:103792.

51. Toğaçar M, Ergen B, Cömert Z. COVID-19 detection using deep learning models to exploit Social Mimic Optimization and structured chest X-ray images using fuzzy color and stacking approaches. Comput Biol Med. 2020;121:103805.
52. Panwar H, Gupta P, Siddiqui MK, Morales-Menendez R, Singh V. Application of deep learning for fast detection of COVID-19 in X-Rays using nCOVnet. Chaos Solit Fractals. 2020;138:109944.

53. Pereira RM, Bertolini D, Teixeira LO, Silla CN Jr, Costa YM. COVID-19 identification in chest X-ray images on flat and hierarchical classification scenarios. Comput Methods Programs Biomed. 2020;194:105532.

54. Waheed A, et al. CovidGAN: Data Augmentation Using Auxiliary Classifier GAN for Improved Covid-19 Detection. IEEE Access. 2020;8:91916-233.

55. Abdel-Basset M, Mohamed R, Elhoseny M, Chakrabortty RK, Ryan M. A Hybrid COVID-19 Detection Model Using an Improved Marine Predators Algorithm and a Ranking-Based Diversity Reduction Strategy. IEEE Access. 2020;8:79521-40.

56. Oh Y, Park S, Ye JC. Deep Learning COVID-19 Features on CXR using Limited Training Data Sets. IEEE Trans Med Imaging. 2020;39(8):2688-700.

57. Vaid S, Kalantar R, Bhandari M. Deep learning COVID-19 detection bias: accuracy through artificial intelligence. Int Orthop. 2020;44:1539-42.

58. Mahmud T, Rahman MA, Fattah SA. CovXNet: A multi-dilation convolutional neural network for automatic COVID-19 and other pneumonia detection from chest X-ray images with transferable multi-receptive feature optimization. Comput Biol Med. 2020;122:103869.

59. Lundh F, Clark A. PILLOW;. Available at https://pillow. readthedocs.io/en/3.1.x/reference/Image.html (2020/05/20).

60. Satapathy S, Naik A. Social group optimization (SGO): a new population evolutionary optimization technique. Complex Intell Syst. 2016;2:173-203.

61. Gupta N, Gupta D, Khanna A, Rebouças Filho PP, de Albuquerque VHC. Evolutionary algorithms for automatic lung disease detection. Measurement. 2019;140:590-608.

62. Kumar S, Sharma B, Sharma VK, Poonia RC. Automated soil prediction using bag-of-features and chaotic spider monkey optimization algorithm. Evol Intell. 2018. https://doi.org/10.1007/ s12065-018-0186-9. 